Canadian

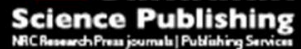

Canadian Journal of Physiology and Pharmacology Revue canadienne de physiologie et pharmacologie

\title{
Reduced expression of HCN channels in sinoatrial node of streptozotocin-induced diabetic rats
}

\begin{tabular}{|r|l|}
\hline Journal: & Canadian Journal of Physiology and Pharmacology \\
\hline Manuscript ID & cjpp-2016-0418.R2 \\
\hline Manuscript Type: & Article \\
\hline Complete List of Authors: & $\begin{array}{l}\text { Huang, Xin; Department of Cardiology, First Affiliated Hospital of Xi'an } \\
\text { Jiaotong University } \\
\text { Zhong, Nier; Department of Cardiology, Shaanxi Provincial People's } \\
\text { Hospital } \\
\text { Zhang, Hong; School of Electrical Engineering, Xi'an Jiaotong University } \\
\text { Ma, Aiqun; Department of Cardiology, First Affiliated Hospital of Xi'an } \\
\text { Jiaotong University } \\
\text { Yuan, Zuyi; Department of Cardiology, First Affiliated Hospital of Xi'an } \\
\text { Jiaotong University } \\
\text { Guo, Ning; Department of Cardiology, First Affiliated Hospital of Xi'an } \\
\text { Jiaotong University }\end{array}$ \\
\hline Keyword: & $\begin{array}{l}\text { streptozotocin-induced diabetes, sinoatrial node dysfunction, HCN } \\
\text { channels, sinoatrial node }\end{array}$ \\
\hline \multicolumn{2}{|c}{} \\
\hline
\end{tabular}


1

2

3

4

5

6

$7 \quad$ Xi'an, Shaanxi, P.R.China, 710061

$8{ }^{2}$ Department of Cardiology, Shaanxi Provincial People's Hospital, Xi'an, Shaanxi,

$9 \quad$ P.R.China, 710068

10

\section{Reduced expression of HCN channels in sinoatrial node of} streptozotocin-induced diabetic rats

${ }^{1}$ Department of Cardiology, First Affiliated Hospital of Xi'an Jiaotong University,

${ }^{3}$ School of Electrical Engineering, Xi'an Jiaotong University, Xi'an, Shaanxi, P.R.China, 710049

Running title: Expression of HCN channels in diabetic rat SAN

\section{*Corresponding Author:}

Prof. Ning Guo, M.D., Ph.D.

Department of Cardiology, First Affiliated Hospital of Xi'an Jiaotong University, No.277 Yanta West Road, Xi'an, Shaanxi, P.R.China, 710061

\section{E-mail: drningguo@163.com}

Conflict of interest: None. 
1 Abstract

2 Diabetes mellitus (DM) is associated with an electrical remodeling of the heart,

3 increasing risk of arrhythmias. However, knowledge of electrical remodeling in

4 sinoatrial node (SAN) by DM is limited. We investigated the expression of HCN

5 channel isoforms, HCN1-HCN4, in SAN from streptozotocin (STZ)-induced diabetic

6 rats and the age-matched controls. We found that the STZ-induced diabetic rats have a

7 lower intrinsic heart rate, a lengthened sinoatrial conduction time and rate-corrected

8 maximal sinoatrial node recovery time in vivo as well as a longer cycle length (CL) in

9 vitro as compared with the control. Optical mapping of the SAN demonstrated an

10 inferior leading pacemaker site, a reduced SAN conduction velocity and diastolic

11 depolarization slope, a longer action potential duration in the STZ-induced diabetic

12 rats than the control. The transcripts and proteins of $\mathrm{HCN} 2$ and $\mathrm{HCN} 4$ in diabetic

13 SAN were reduced. Specific blockade of $\mathrm{HCN}$ channels by $3 \mathrm{uM}$ ivabradine 14 significantly prolonged CL of Langendorff heart by $18 \%$ in the diabetic rats and $26 \%$

15 in the control. The reduced expression of $\mathrm{HCN}$ channel isoforms in SAN of the 16 STZ-induced diabetic rat may be an important contributor to the reduced SAN 17 function in DM.

19 Keywords:

20 streptozotocin-induced diabetes, sinoatrial node dysfunction, HCN channels, 21 sinoatrial node. 


\section{Introduction}

2 Diabetes mellitus (DM) is associated with increased risk of arrhythmias, which may

3 occur in the absence of atherosclerosis, hypertension or valvular dysfunction (Gami et

4 al. 2007). Ventricular arrhythmias are the most frequent type of arrhythmia observed

5 in DM patients (Veglio et al. 2000). Electrophysiological studies have shown that the

6 action potential (AP) duration (APD) of ventricular myocytes was prolonged and the

7 transient outward $\mathrm{K}^{+}$current $\left(\mathrm{I}_{\mathrm{to}}\right)$, L-type $\mathrm{Ca}^{2+}$ current $\left(\mathrm{I}_{\mathrm{CaL}}\right)$, and inward rectifier

$8 \mathrm{~K}^{+}$current $\left(\mathrm{I}_{\mathrm{K} 1}\right)$ were reduced in type 1 and 2 diabetic models from a variety of

9 species (Lengyel et al. 2007; Tsuchida and Watajima 1997; Wang et al. 1995).

Expression of the channel pore-forming subunits also decreased in the diabetic heart (Nishiyama et al. 2001; Qin et al. 2001). All these findings revealed an electrical remodeling in the diabetic heart. However, knowledge of electrical remodeling in SAN by DM is very limited.

Sinoatrial node dysfunction (SND), either tachycardia or bradycardia, is often seen in both type 1 and type 2 DM (Linnemann and Janka 2003). A reduced spontaneous pacemaking rate of the isolated heart and right atrial preparation was observed in streptozotocin (STZ)-induced DM animal model with a lengthened APD of SAN, which suggested a reduced SAN function (Howarth et al. 2007; Kofo-Abayomi and Lucas 1988). However, the underlying molecular mechanism of SAN electrical remodeling associated with DM remains uncertain.

It is evidenced that the pacemaker current $\left(\mathrm{I}_{\mathrm{f}}\right)$ carried by the hyperpolarization-activated cyclic nucleotide-gated $(\mathrm{HCN})$ cation channels plays an 
1 important role in pacemaking and heart rate control. Mutations of HCN channel genes

2 were found to be associated with congenital SND (Milanesi et al. 2006). So far, four

$3 \mathrm{HCN}$ channel isoforms have been identified in mammal, that is HCN1-HCN4

4 (Ludwig et al. 1998). Considering the vital significance of HCN channels in

5 pacemaking, we hypothesized the altered expression of HCN channel isoforms may

6 be one of the molecular mechanisms of SND during DM.

\section{$7 \quad$ Materials and methods}

8

9

\section{Animal preparation}

DM was induced by a single intravenous injection of STZ (65 mg/kg; Sigma, USA) through tail vein to Sprague-Dawley rats (200-250 g, 3 months old). After $72 \mathrm{~h}$ and overnight fasting, blood samples were obtained from the tail vein and the glucose concentration was measured. Animals with fasting blood glucose levels on above 250 $\mathrm{mg} / \mathrm{dl}$ were considered as diabetic. In this experiment, 35 out of 40 rats were modeled successfully. Age-matched controls received an equivalent volume of the citrate buffer solution alone. All animals were maintained on the same diet and water ad libitum for 20 weeks. Non-fasting blood glucose level was measured every 4 weeks at $11 \mathrm{am}$. Blood was obtained from a tail vein, and glucose concentrations were determined using an Accu-Chek Performa glucose meter (Roche Diagnostics, Switzerland).

All animal procedures were performed in accordance with the Guide for the Care and Use of Laboratory Animals issued by the Institute of Laboratory Animal Resources (NIH publication 85-23, revised 1996) and approved by the Xi'an Jiaotong University Animal Care and Use Committee. 
1

2 Six-lead ECGs were recorded under anesthesia with sodium pentobarbitone (40

\section{Sinoatrial node function} $\mathrm{mg} / \mathrm{kg}$, i.p, Sigma, USA) from four leads of rats. The stimuli were applied to the right

4

atrial appendage by a bipolar stimulus through esophagus. Calculated sinoatrial conduction time (SACT) was tested by a train of 8-10 stimuli at a rate of 10-20 bpm above the baseline heart rate and was repeated three times (Narula et al. 1978). For each rat, three SACT values were averaged. The sinus node recovery time (SNRT) was obtained by overdrive pacing for $30 \mathrm{sec}$, beginning at a frequency of $6.5 \mathrm{~Hz}$ with $0.5 \mathrm{~Hz}$ increment until the SNRT was no longer lengthened (Breithardt et al. 1977). The longest time interval from the last paced atrial depolarization to the first spontaneous sinus cycle was designated as the SNRTmax. The rate-corrected maximal sinoatrial node recovery time (CSNRTmax) was determined by subtracting the baseline sinus cycle length (CL) from the SNRTmax.

\section{The intrinsic heart rate (IHR) in vivo}

The IHR was tested under complete blockade of the cardiac autonomic nervous system with propranolol ( $3 \mathrm{mg} / \mathrm{kg}$ per $\mathrm{ml}$ i.v., Sigma, USA) and atropine (2 mg/kg per $\mathrm{ml}$ i.v., Sigma, USA) as previously reported and averaged for $1 \mathrm{~min}$ (Safa-Tisseront et al. 1998).

\section{The cycle length in Langendorff hearts}

Hearts were excised and perfused retrogradely through aorta on a Langendorff apparatus at a constant pressure of $80 \mathrm{mmHg}$ with warm $\left(36 \pm 0.5^{\circ} \mathrm{C}\right)$, oxygenated, modified Krebs-Henseleit bicarbonate (KHB) buffer as described previously (Maier et 
1 al. 2003). The composition of the modified KHB was as follows: (in $\mathrm{mM}$ ) $\mathrm{NaCl} 118$,

$2 \mathrm{KCl} 4.7, \mathrm{MgSO}_{4} 1.2, \mathrm{KH}_{2} \mathrm{PO}_{4} 1.2$, glucose 11.0, $\mathrm{CaCl}_{2} 2.0$, pyruvate 2.0, $\mathrm{NaHCO}_{3} 25$

3 and insulin $5 \mathrm{U} / \mathrm{L}(\mathrm{PH} 7.4 \pm 0.5)$. Two recording electrodes were attached to the atrial

4 appendages, and data were sampled with a BL-420 Electrophysiolograph (Chengdu

5 Taimeng Technology Co., Ltd, China). Electrocardiographies were recorded at

6 baseline and at the end of 25 min perfusion of $3 \mathrm{uM}$ ivabradine (Sigma, USA) which

7 has previously been demonstrated to reduce $I_{f}$ by $60 \%$ without obvious effect on

8 T-type and L-type calcium current and delayed rectifier potassium current (Bois et al.

9 1996); subsequently, ivabradine was washed out with KHB buffer for 25 min. After

10 perfusion, heart samples were removed, blotted dry and weighed. The CL was

11 averaged over $3 \mathrm{~min}$.

\section{Laser capture microdissection (LCM)}

The HE-stained, dehydrated tissue section was overlaid with a thermoplastic transfer film mounted on an optically transparent CapSure HS cap (Arcturus, USA). Histologically, the SAN is seen as a dense aggregation of specialized cells within a highly fibrous connective tissue matrix (Hudson 1960). With HE-staining, SAN cells are small interlacing cells, less darkly stained due to their poorly developed microfilament cytoskeleton, with an appearance distinct from neighboring atrial myocytes (James et al. 1966). The SAN cells with above typical light microscope characteristics were dissected and affixed to the film by laser pulses from the PixCell II LCM System (Arcturus, USA). The LCM System was set to the following parameters: 7-7.5 $\mu \mathrm{m}$ laser spot size, $60 \mathrm{~mW}$ power, 2.5-3.0 ms duration. 


\section{Real-time quantitative reverse transcription-polymerase chain reaction}

2 The first-strand cDNAs were synthesized and amplified in real-time quantitative

3 reverse transcription-polymerase chain reactions (PCR) using the Super-Script ${ }^{\mathrm{TM}}$ III

$4 \quad$ Platinum $^{\circledR}$ CellsDirect Two-Step qRT-PCRKit with SYBR ${ }^{\circledR}$ Green (Invitrogen, USA).

5 The internal control was $\beta$-actin. Table 1 lists the primer pair sequences used in PCR.

6 Real-time PCR reactions were performed in duplicate using the ABI PRISM 7000

7 (Applied Biosystems, USA) with the following cycling programme: one step at $50{ }^{\circ} \mathrm{C}$

8 for $2 \mathrm{~min}$, one step at $95{ }^{\circ} \mathrm{C}$ for $2 \mathrm{~min}$, and 45 cycles at $95{ }^{\circ} \mathrm{C}$ for $15 \mathrm{sec}$ and $60{ }^{\circ} \mathrm{C}$ for

$930 \mathrm{sec}$. The data were analyzed using the $2^{-\Delta \Delta \mathrm{CT}}$ method (Livak and Schmittgen

10 2001).

11

\section{Immunohistochemistry}

The intact SAN preparation was isolated, fixed, dehydrated and embedded in optimal cutting temperature (OCT) compound (Tissue-Tek, NL). Frozen $10-\mu \mathrm{m}$ sections were serially cut across the leading pacemaker site perpendicular to the crista terminalis. Serial sections were labeled with rabbit polyclonal antibodies against connexin 43 (Cx43) (1:2000; Sigma, USA) and the hyperpolarization-activated cyclic nucleotide-gated potassium channel 1-4 (HCN1-4) (1:200; Alomone Labs, Israel), respectively. The FITC-conjugated goat anti-rabbit IgG (Zhongshan Golden Bridge Biotechnology Corporation, China) was used as the secondary antibody with a dilution factor of 1:100. For positive control experiments, rat brain tissue was used. For the negative control experiments, the primary antibody was substituted by horse serum (4\%) alone. The sections were analyzed under a Leica TCS SP 2 laser scanning 
1 confocal microscope (Leica, Germany).

\section{Optical mapping}

3 The high resolution optical mapping was used to investigate the pacemaking activities

4 and the patterns of electrical conduction in the atrial preparations containing the SAN

5 as described previously (Huang et al. 2007). Briefly, the atrial preparations were

6 stained by superfusion with a bolus of $2 \mathrm{mM}$ di-4-ANEPPS (Molecular Probes, USA)

7 and fixed in a chamber with the endocardial side toward the high-speed

8 charge-coupled device camera (CA-D1-0128T, Dalsa, Canada). The voltage-sensitive

9 fluorescent signals were recorded from $64 \times 64$ sites simultaneously at a rate of 910

frames/s. The location of the leading pacemaker site was assessed in all hearts. Shifts in leading pacemaker site were quantified using a grid in which the vertical axis was placed parallel, and just adjacent to the crista terminalis. The horizontal axis was placed along the edge of the opening of the inferior vena cava, perpendicular to the vertical axis. All optical data were analyzed using custom software written in Matlab. Analyses included pseudocolour electrical activation maps and isochronal contour plots, which were generated from measurements of activation time at individual pixels. In all cases background fluorescence was subtracted. The optical action potential was generated by measuring the change in fluorescence as a function of time at the leading pacemaker of the SAN. APD and diastolic depolarization (DD) slope measurements were averages from 3-4 consecutive optical action potentials in one preparation. DD slope was measured by fitting this phase with a straight line and determining its slope, which we quantified as a relative change in fluorescence units perms ( $\Delta \mathrm{F} / \mathrm{ms})$. Local 
1 conduction velocity (CV) was quantified specifically in the leading pacemaker site

2 using an established approach previously described (Nygren et al. 2004). Briefly,

3 activation times at each pixel from a $7 \times 7$ pixel array were determined and fit to a

4 plane using the least squares fit method. The direction on this plane that is increasing

5 the fastest represents the direction that is perpendicular to the wave front of electrical

6 propagation and the maximal slope represents the inverse of the speed of conduction

7 in that direction.

8 Western blot assay

9 Membrane proteins were extracted from the leading pacemaker site of SAN, by centrifugation on a sucrose buffer (including protease inhibitor cocktail) according to the protocol published previously (Jia et al. 2014). The rabbit polyclonal antibodies to rat HCN2 and HCN4 (1:200 dilution, respectively) or mouse monoclonal antibody to rat $\beta$-actin (1:2000 dilution, Santa Cruz Biotechnology Inc., USA) were used as the primary antibodies. The goat anti-rabbit IRDye ${ }^{\circledR} 800 \mathrm{CW}$ or goat anti-mouse IRDye ${ }^{\circledR}$ 680 LT secondary antibody (1:2000 dilution) (Li-COR Biosciences, USA) were used as the secondary antibodies. Quantitative analysis of the western blot results was performed with the Odyssey software 3.0 (Li-COR Bioscience, USA). For positive control experiments, membrane proteins of rat brain tissue were used. For the negative control experiments, the primary antibody was substituted by phosphate buffered saline (PBS) alone.

\section{Statistical analysis}

Data are expressed as the means \pm SEM. The significance of difference was 
1 determined by analysis of variance (ANOVA) and a post hoc multiple comparison test

2 using the SPSS 11. 0 software. Statistical significance was established at $p<0.05$.

$3 \quad$ Results

$4 \quad$ Physical characteristics of diabetic rats

5 As illustrated in Figure 1, the resting heart rate (RHR) and IHR of the rats injected

6 with STZ declined continuously within the first 8 weeks after injection and then

7 maintained throughout the observation period, while the control arm was relatively

8 stable. In contrast, blood glucose level of STZ-induced rats elevated continuously

9 within the first 8 weeks after STZ injection and then maintained throughout the observation period. The STZ-induced diabetic rats have higher body weight, heart weight, heart-to-body weight ratio than the age-matched control group ( $n=10$ for each group, each $p<0.05$ ). The SACT and CSNRTmax of the diabetic rats were significantly lengthened as compared to the control $(n=10$ for each group, each $p<$ $0.05)$.

\section{Effect of ivabradine on the CL in Langendorff hearts}

As illustrated in Figure 2A, the CL of Langendorff hearts from the STZ-induced diabetic rats was longer than that of the age-matched controls ( $316 \pm 5 \mathrm{~ms}$ vs. $247 \pm 7$ ms, $n=5, p<0.05)$. Specific blockade of HCN channels by 3 uM ivabradine prolonged CL by $26 \%$ (from $247 \pm 7 \mathrm{~ms}$ to $310 \pm 8 \mathrm{~ms}$ ) in the control but $18 \%$ (from $316 \pm 5 \mathrm{~ms}$ to $372 \pm 12 \mathrm{~ms}$ ) in the diabetic rats. As compared with the control, the pacemaking activity of the diabetic hearts is less sensitive to ivabradine ( $n=5, p<$ 0.05) (Figure 2B). 


\section{Transcription of $\mathrm{HCN}$ channel isoforms in SAN}

2 The cells with typical light microscope characteristics of the SAN cells were dissected

3 specifically by LCM for further analysis (Figure 3A, B and C). The transcripts of

$4 \mathrm{HCN} 2$ and $\mathrm{HCN} 4$ were abundant in the rat SAN, while neither the HCN1 transcript

5 nor HCN3 was detectable (data not shown). The transcripts of HCN2 and HCN4 in

6 SAN were reduced in the STZ-induced diabetic rats as compared with the control

7 (HCN2: $3.41 \pm 0.32$ in the diabetic rats vs. $4.13 \pm 0.15$ in the control, $p=0.018, n=5$

8 for each group; $H C N 4: 2.14 \pm 0.23$ in the diabetic rats vs. $3.40 \pm 0.50$ in the control; $p$

$9 \quad=0.038 ; n=5$ for each group) (Figure 3D).

\section{Pacemaking activity and activation pattern in SAN}

Pacemaker activities and activation patterns were determined from voltage-sensitive fluorescent signals recorded over the SAN and surrounding atrial muscle (Figure 4A). Representative activation maps for STZ-induced diabetic rats and the control (Figure 4B and $4 C$ ) in sinus rhythm demonstrate that the STZ-induced diabetic rats had an inferior leading pacemaker site adjacent to the crista terminalis within the right atrial posterior wall and a slower conduction time than the control which had a leading pacemaker site in the superior region of the right atrial posterior wall near the opening of the superior vena cava. Beat to beat variability in the location of the leading pacemaker site within the same heart was not observed.

Cycle length was longer (corresponding to a lower pacemaking rate) in STZ-induced diabetic rats compared to the control $(361 \pm 4 \mathrm{~ms}$ vs. $269 \pm 5 \mathrm{~ms}, n=20, p<0.01$;

Figure 4D). SAN CV was lower in STZ-induced diabetic hearts compared to the 
1 controls $(4.8 \pm 0.2 \mathrm{~cm} / \mathrm{s}$ vs. $5.9 \pm 0.1 \mathrm{~cm} / \mathrm{s}, n=20, p<0.05$; Figure $4 \mathrm{E})$.

2 Figure 4F shows representative optical APs from SAN. Quantitative analysis

3 demonstrates that the DD slope was reduced in the SAN of diabetic rats $(0.17 \pm 0.02 \Delta$

$4 \mathrm{~F} / \mathrm{ms}$ vs. $0.21 \pm 0.01 \Delta \mathrm{F} / \mathrm{ms}, n=20, p<0.05$; Figure $4 \mathrm{G}$ ). APD at $50 \%$ and $70 \%$ in

5 SAN were $55.2 \pm 3.2$ and $63.5 \pm 3.4 \mathrm{~ms}$ in diabetic rat heart compared to $44.0 \pm 2.1$

6 and $51.5 \pm 2.6 \mathrm{~ms}$ in controls $(n=20$, each $p<0.05$; Figure $4 \mathbf{H})$.

\section{Expression of $\mathrm{HCN}$ channel isoform proteins in SAN}

8 As illustrated in Figure 5A, SAN cells lack connexin 43 (Cx43), instead, adjacent

9 atrial working myocytes take on positive staining of $\mathrm{Cx} 43$. The SAN tissues have robust $\mathrm{HCN} 2$ and $\mathrm{HCN} 4$ staining with negative $\mathrm{HCN} 1$ and $\mathrm{HCN} 3$ staining (data not shown).

Only the leading pacemaker site identified by optical mapping was harvested for further Western blot assay. As shown in Figure 5B, the protein expression of HCN2 was reduced by $70 \%$ from $0.56 \pm 0.06$ in the control to $0.17 \pm 0.05$ in the STZ-induced diabetic rats ( $p<0.01 ; n=20$ for each group). And HCN4 protein was reduced by $58 \%$ by STZ (HCN4: $0.21 \pm 0.04$ in the diabetic rats vs. $0.50 \pm 0.02$ in the control; each $p<0.01 ; n=20$ for each group).

\section{Discussion}

The main findings of the present study is that (1) reduced SAN function in STZ-induced diabetic rats; (2) reduced contribution of HCN channel isoforms (HCN2 and $\mathrm{HCN} 4$ ) to automaticity of SAN; (3) and its down-regulating expression during DM. 
$2 \mathrm{DM}$ is a serious public health concern with the estimated global prevalence of $4.4 \%$ in 32030 (Wild et al. 2004). SND is often seen in both type 1 and type 2 DM (Linnemann

4 and Janka 2003). The STZ-induced diabetic rat is a widely used experimental model

5 of type 1 DM. Its general characteristics include hyperglycaemia, hypoinsulinaemia

6 and hyperlipidaemia (Dai et al. 1994). In our study, experiments were performed 20

7 weeks after STZ-treatment. The general characteristics of the diabetic rats compared

8 with the age-matched controls included (1) a significant increase in non-fasting blood

9 glucose; (2) lengthened SACT as well as CSNRTmax, lower RHR and IHR in vivo and a longer CL in vitro, (3) an inferior leading pacemaker site, a reduced SAN conduction velocity, a reduced DD slope and prolonger APD, these indicated an impaired SAN function in the STZ-induced diabetic rats. In accordance with our findings, in vivo biotelemetry studies revealed that the heart rate is progressively and rapidly reduced after administration of STZ and the reduction in heart rate can be partially normalized with insulin replacement (Howarth et al. 2006). Further studies demonstrated that the spontaneous beating rate of the Langendorff heart was significantly reduced and APD at $50 \%$ and $70 \%$ from peak AP were significantly prolonged in SAN from STZ-induced diabetic rat (Hicks et al. 1998; Howarth et al. 2007; Zhang et al. 2002). Some studies also found that mice with STZ-induced type 1 DM manifested a reduced RHR, prolonged CSNRT and an attenuated chronotropic response to isoprenaline in isolated, perfused hearts (Luo et al. 2013). Similar SND is present in Leptin-receptor deficient homozygous $d b / d b$ mouse, a model of type $2 \mathrm{DM}$ 
1 (Soltysinska et al. 2014). In addition, the abnormalities of automaticity and

2 conduction were observed in alloxan-diabetic rabbit model, including lower sinus rate,

3 longer SNRT, inhomogeneity of atrial conduction and the occurrence of

4 atrioventricular block (Senges et al. 1980). Therefore, DM may alter the

5 electrophysiological property of SAN, and defects in the expression and/or function of

6 ion channels might underlie heart rhythm disturbances in DM.

\section{HCN channel and SAN function}

8 The HCN channels activate early in phase 4 when the cell is hyperpolarized and thus

9 contribute to early diastolic depolarization. The mammalian genome encodes four

$\mathrm{HCN}$ channels, that is $\mathrm{HCN} 1-\mathrm{HCN} 4$, which share $\sim 80 \%$ of homology but distinct biophysical properties and expression profiles (Biel et al. 2002; Clapham 1998; Kaupp and Seifert 2001; Ludwig et al. 1998; Stieber et al. 2004). HCN4 is the dominant $\mathrm{HCN}$ isoform in adult SAN of rabbit, murine and canine, accounting for $80 \%$ of the total HCN transcript (Moosmang et al. 2001; Shi et al. 1999). HCN4 knock-out mice demonstrated $50 \%$ reduction in heart rate (Baruscotti et al. 2011), or recurrent sinus pauses (Herrmann et al. 2007). HCN1 takes up $18 \%$ of the total $\mathrm{HCN}$ mRNA in rabbit SAN but only at very low level in mouse SAN (Moosmang et al. 2001). The main cardiac phenotype of the HCN1 knock-out mice is a pronounced SND characterized by impaired impulse formation and sinoatrial conduction, resulting in bradycardia, sinus arrhythmia, and recurrent sinus pauses (Fenske et al. 2013). The HCN2 transcripts are also expressed in mouse SAN. However, they are equally distributed among atria and SAN at a low to moderate level (Moosmang et al. 2001). 
1 HCN2-null mice also displayed cardiac sinus dysrhythmia, a shift of the maximum

2 diastolic potential to hyperpolarized values. Mice with cardiomyocyte-specific

3 deletion of HCN2 displayed the same dysrhythmia as mice lacking HCN2 globally,

4 indicating that the dysrhythmia is indeed caused by dysfunction of SAN (Ludwig et al.

5 2003). By contrast, $H C N 3$-deficient mice display normal cardiac pacemaker activity

6 with an abnormality in the ventricular repolarization (Fenske et al. 2011).

7 The expression HCN channel isoforms is dynamic during different physiological and

8 pathophysiological conditions. Our previous study revealed that the age-associated

9 impairment of SAN function was accompanied with a reduced expression of HCN channels (Huang et al. 2007; Huang et al. 2016). In spontaneously beating fetal and neonatal ventricular myocytes, a prominent $I_{f}$ is detectable. During maturation, $I_{f}$ density decreases, and ventricular myocytes lose their ability to generate spontaneous action potentials (Cerbai et al. 1999; Robinson et al. 1997). Re-expression of $I_{\mathrm{f}}$ in adult ventricular myocytes was also found in ventricular hypertrophy and heart failure (Cerbai et al. 1996; Fernandez-Velasco et al. 2003). This disease-dependent alteration of $\mathrm{I}_{\mathrm{f}}$ is mirrored by an increased expression of HCN channels. Examination of mRNA levels in hypertrophied ventricular myocytes revealed an upregulation of HCN2 and HCN4 in different animal models (Fernandez-Velasco et al. 2003). In addition, analysis of human myocytes obtained from patients with end-stage heart failure shows an increase in HCN4 mRNA (Borlak and Thum 2003). It was observed that the upregulation of $I_{f}$ in diseased myocytes results in the occurrence of a diastolic depolarization in ventricular myocytes (Sridhar et al. 2006). Experiments with 
1 adenoviral HCN2 constructs support these finding. Infected myocytes develop a

2 pronounced phase 4 depolarization and spontaneous activity as a result of HCN2

3 overexpression ( $\mathrm{Qu}$ et al. 2001). Taken together, HCN channels may contribute

4 significantly to the formation of arrhythmias in certain cardiac disease states, leading

5 to the hypothesis that an altered expression of $\mathrm{HCN}$ channel isoforms may trigger, at

6 least in part, the declined function of diabetic SAN.

\section{Reduced expression of HCN channel isoforms in diabetic SAN}

8 This study indicated that in the STZ-induced diabetic rat, the expression of HCN2 and

9 HCN4 in SAN was reduced at both transcription and protein levels. In order to correlate the expression and function, a blocker of HCN channels, ivabradine was used to perfuse in Langendorff hearts. Ivabradine is not an isoform-specific blocker. Experiments investigating the affinity of ivabradine for homomeric $\mathrm{HCN}$ channels do not reveal any substantial isoform-specificity. Ivabradine blocked the slow inward current through expressed human $\mathrm{HCN} 1, \mathrm{HCN} 2, \mathrm{HCN} 3$, and $\mathrm{HCN} 4$ channels with mean half maximal inhibitory concentration of $2.25 \mathrm{uM}$ (Stieber et al. 2006). Although our study could not define the specific contribution of each $\mathrm{HCN}$ isoform, the prolonged CL led by ivabradine perfusion at least suggests that blockade of HCN channels contributes to the reduction in spontaneous pacemaking rate. The reduced effect of ivabradine in the STZ-induced diabetic rat heart in turn may be caused by reduced expression of $\mathrm{HCN}$ channels.

In summary, the expression of $\mathrm{HCN}$ channel isoforms in SAN was reduced in STZ-induced diabetic rat, which may be an important contributor to the reduced SAN 
3

4

function in DM. This needs further functional test by electrophysiology to verify.

\section{Acknowledgements}

This work was supported by grants from The National Science Foundation of China (NSFC) (No. 81100132, 81170138), the Fundamental Research Funds for the Central Universities (No. 1191320117) and the First Affiliated Hospital of XJTU Fund (No. 2015yk26).

\section{References:}

Baruscotti, M., Bucchi, A., Viscomi, C., Mandelli, G., Consalez, G., Gnecchi-Rusconi, T., Montano, N., Casali, K.R., Micheloni, S., Barbuti, A., and DiFrancesco, D. 2011. Deep bradycardia and heart block caused by inducible cardiac-specific knockout of the pacemaker channel gene Hcn4. Proc. Natl. Acad. Sci. U S A. 108(4): 1705-1710. doi: $10.1073 /$ pnas. 1010122108 .

Biel, M., Schneider, A., and Wahl, C. 2002. Cardiac HCN channels: structure, function, and modulation. Trends. Cardiovasc. Med. 12(5): 206-212.

Bois, P., Bescond, J., Renaudon, B., and Lenfant, J. 1996. Mode of action of bradycardic agent, S 16257, on ionic currents of rabbit sinoatrial node cells. Br. J. Pharmacol. 118(4): 1051-1057.

Borlak, J., and Thum, T. 2003. Hallmarks of ion channel gene expression in end-stage heart failure. FASEB. J. 17(12): 1592-1608. doi: 10.1096/fj.02-0889com.

Breithardt, G., Seipel, L., and Loogen, F. 1977. Sinus node recovery time and calculated sinoatrial conduction time in normal subjects and patients with sinus node dysfunction. Circulation. 56(1): 
1

2

3

4

5

6

7

$43-50$.

Cerbai, E., Barbieri, M., and Mugelli, A. 1996. Occurrence and properties of the hyperpolarization-activated current If in ventricular myocytes from normotensive and hypertensive rats during aging. Circulation. 94(7): 1674-1681.

Cerbai, E., Pino, R., Sartiani, L., and Mugelli, A. 1999. Influence of postnatal-development on I(f) occurrence and properties in neonatal rat ventricular myocytes. Cardiovasc. Res. 42(2): 416-423.

Clapham, D.E. 1998. Not so funny anymore: pacing channels are cloned. Neuron. 21(1): 5-7.

Dai, S., Thompson, K.H., and McNeill, J.H. 1994. One-year treatment of streptozotocin-induced diabetic rats with vanadyl sulphate. Pharmacol. Toxicol. 74(2): 101-109.

Fenske, S., Krause, S.C., Hassan, S.I., Becirovic, E., Auer, F., Bernard, R., Kupatt, C., Lange, P., Ziegler, T., Wotjak, C.T., Zhang, H., Hammelmann, V., Paparizos, C., Biel, M., and Wahl-Schott, C.A. 2013. Sick sinus syndrome in HCN1-deficient mice. Circulation. 128(24): 2585-2594. doi: 10.1161/CIRCULATIONAHA.113.003712.

Fenske, S., Mader, R., Scharr, A., Paparizos, C., Cao-Ehlker, X., Michalakis, S., Shaltiel, L., Weidinger, M., Stieber, J., Feil, S., Feil, R., Hofmann, F., Wahl-Schott, C., and Biel, M. 2011. HCN3 contributes to the ventricular action potential waveform in the murine heart. Circ. Res. 109(9): 1015-1023. doi: 10.1161/CIRCRESAHA.111.246173.

Fernandez-Velasco, M., Goren, N., Benito, G., Blanco-Rivero, J., Bosca, L., and Delgado, C. 2003.

Regional distribution of hyperpolarization-activated current (If) and hyperpolarization-activated cyclic nucleotide-gated channel mRNA expression in ventricular cells from control and hypertrophied rat hearts. J. Physiol. 553(Pt 2): 395-405. doi: 10.1113/jphysiol.2003.041954.

Gami, A.S., Witt, B.J., Howard, D.E., Erwin, P.J., Gami, L.A., Somers, V.K., and Montori, V.M. 
1 2007. Metabolic syndrome and risk of incident cardiovascular events and death: a systematic

2 review and meta-analysis of longitudinal studies. J. Am. Coll. Cardiol. 49(4): 403-414. doi:

$3 \quad 10.1016 /$ j.jacc.2006.09.032.

4 Herrmann, S., Stieber, J., Stockl, G., Hofmann, F., and Ludwig, A. 2007. HCN4 provides a

5 'depolarization reserve' and is not required for heart rate acceleration in mice. EMBO. J. 26(21):

$6 \quad$ 4423-4432. doi: 10.1038/sj.emboj.7601868.

7 Hicks, K.K., Seifen, E., Stimers, J.R., and Kennedy, R.H. 1998. Effects of streptozotocin-induced

8 diabetes on heart rate, blood pressure and cardiac autonomic nervous control. J. Auton. Nerv. Syst.

9 69(1): 21-30.

10

11

12

Howarth, F.C., Al-Sharhan, R., Al-Hammadi, A., and Qureshi, M.A. 2007. Effects of streptozotocin-induced diabetes on action potentials in the sinoatrial node compared with other regions of the rat heart. Mol. Cell. Biochem. 300(1-2): 39-46. doi: 10.1007/s11010-006-9366-5.

Howarth, F.C., Jacobson, M., Shafiullah, M., and Adeghate, E. 2006. Effects of insulin treatment on heart rhythm, body temperature and physical activity in streptozotocin-induced diabetic rat. Clin. Exp. Pharmacol. Physiol. 33(4): 327-331. doi: 10.1111/j.1440-1681.2006.04370.x.

Huang, X., Yang, P., Du, Y., Zhang, J., and Ma, A. 2007. Age-related down-regulation of HCN channels in rat sinoatrial node. Basic. Res. Cardiol. 102(5): 429-435. doi: $10.1007 / \mathrm{s} 00395-007-0660-5$.

Huang, X., Yang, P., Yang, Z., Zhang, H., and Ma, A. 2016. Age-associated expression of HCN channel isoforms in rat sinoatrial node. Exp. Biol. Med. (Maywood). 241(3): 331-339. doi: $10.1177 / 1535370215603515$.

Hudson, R.E. 1960. The human pacemaker and its pathology. Br. Heart. J. 22: 153-167. 
1 James, T.N., Sherf, L., Fine, G., and Morales, A.R. 1966. Comparative ultrastructure of the sinus

2 node in man and dog. Circulation. 34(1): 139-163.

3 Jia, B., Choy, E., Cote, G., Harmon, D., Ye, S., Kan, Q., Mankin, H., Hornicek, F., and Duan, Z.

4 2014. Cyclin-dependent kinase 11 (CDK11) is crucial in the growth of liposarcoma cells. Cancer.

$5 \quad$ Lett. 342(1): 104-112. doi: 10.1016/j.canlet.2013.08.040.

6 Kaupp, U.B., and Seifert, R. 2001. Molecular diversity of pacemaker ion channels. Annu. Rev.

7 Physiol. 63: 235-257. doi: 10.1146/annurev.physiol.63.1.235.

8 Kofo-Abayomi, A., and Lucas, P.D. 1988. A comparison between atria from control and

9 streptozotocin-diabetic rats: the effects of dietary myoinositol. Br. J. Pharmacol. 93(1): 3-8.

Lengyel, C., Virag, L., Biro, T., Jost, N., Magyar, J., Biliczki, P., Kocsis, E., Skoumal, R., Nanasi,

P.P., Toth, M., Kecskemeti, V., Papp, J.G., and Varro, A. 2007. Diabetes mellitus attenuates the

repolarization reserve in mammalian heart. Cardiovasc. Res. 73(3): 512-520. doi: 10.1016/j.cardiores.2006.11.010.

Linnemann, B., and Janka, H.U. 2003. Prolonged QTc interval and elevated heart rate identify the

type 2 diabetic patient at high risk for cardiovascular death. The Bremen Diabetes Study. Exp. Clin.

Endocrinol. Diabetes. 111(4): 215-222. doi: 10.1055/s-2003-40466.

Livak, K.J., and Schmittgen, T.D. 2001. Analysis of relative gene expression data using real-time

quantitative PCR and the 2(-Delta Delta C(T)) Method. Methods. 25(4): 402-408. doi:

Ludwig, A., Budde, T., Stieber, J., Moosmang, S., Wahl, C., Holthoff, K., Langebartels, A.,

Wotjak, C., Munsch, T., Zong, X., Feil, S., Feil, R., Lancel, M., Chien, K.R., Konnerth, A., Pape,

H.C., Biel, M., and Hofmann, F. 2003. Absence epilepsy and sinus dysrhythmia in mice lacking 
the pacemaker channel HCN2. EMBO. J. 22(2): 216-224. doi: 10.1093/emboj/cdg032.

Ludwig, A., Zong, X., Jeglitsch, M., Hofmann, F., and Biel, M. 1998. A family of hyperpolarization-activated mammalian cation channels. Nature. 393(6685): 587-591. doi: $10.1038 / 31255$.

Luo, M., Guan, X., Luczak, E.D., Lang, D., Kutschke, W., Gao, Z., Yang, J., Glynn, P., Sossalla, S., Swaminathan, P.D., Weiss, R.M., Yang, B., Rokita, A.G., Maier, L.S., Efimov, I.R., Hund, T.J., and Anderson, M.E. 2013. Diabetes increases mortality after myocardial infarction by oxidizing CaMKII. J. Clin. Invest. 123(3): 1262-1274. doi: 10.1172/JCI65268.

Maier, S.K., Westenbroek, R.E., Yamanushi, T.T., Dobrzynski, H., Boyett, M.R., Catterall, W.A., and Scheuer, T. 2003. An unexpected requirement for brain-type sodium channels for control of heart rate in the mouse sinoatrial node. Proc. Natl. Acad. Sci. U S A. 100(6): 3507-3512. doi: 10.1073/pnas.2627986100.

Milanesi, R., Baruscotti, M., Gnecchi-Ruscone, T., and DiFrancesco, D. 2006. Familial sinus bradycardia associated with a mutation in the cardiac pacemaker channel. N. Engl. J. Med. 354(2): 151-157. doi: 10.1056/NEJMoa052475.

Moosmang, S., Stieber, J., Zong, X., Biel, M., Hofmann, F., and Ludwig, A. 2001. Cellular expression and functional characterization of four hyperpolarization-activated pacemaker channels in cardiac and neuronal tissues. Eur. J. Biochem. 268(6): 1646-1652.

Narula, O.S., Shantha, N., Vasquez, M., Towne, W.D., and Linhart, J.W. 1978. A new method for measurement of sinoatrial conduction time. Circulation. 58(4): 706-714.

Nishiyama, A., Ishii, D.N., Backx, P.H., Pulford, B.E., Birks, B.R., and Tamkun, M.M. 2001. Altered $\mathrm{K}(+)$ channel gene expression in diabetic rat ventricle: isoform switching between $\mathrm{Kv} 4.2$ 
1

2

3

4

5

6

7

8

9

and Kv1.4. Am. J. Physiol. Heart. Circ. Physiol. 281(4): H1800-1807.

Nygren, A., Lomax, A.E., and Giles, W.R. 2004. Heterogeneity of action potential durations in isolated mouse left and right atria recorded using voltage-sensitive dye mapping. Am. J. Physiol. Heart. Circ. Physiol. 287(6): H2634-2643. doi: 10.1152/ajpheart.00380.2004.

Qin, D., Huang, B., Deng, L., El-Adawi, H., Ganguly, K., Sowers, J.R., and El-Sherif, N. 2001.

Downregulation of $\mathrm{K}(+)$ channel genes expression in type I diabetic cardiomyopathy. Biochem. Biophys. Res. Commun. 283(3): 549-553. doi: 10.1006/bbrc.2001.4825.

Qu, J., Barbuti, A., Protas, L., Santoro, B., Cohen, I.S., and Robinson, R.B. 2001. HCN2 overexpression in newborn and adult ventricular myocytes: distinct effects on gating and excitability. Circ. Res. 89(1): E8-14.

Robinson, R.B., Yu, H., Chang, F., and Cohen, I.S. 1997. Developmental change in the voltage-dependence of the pacemaker current, if, in rat ventricle cells. Pflugers. Arch. 433(4): $533-535$.

Safa-Tisseront, V., Ponchon, P., Laude, D., and Elghozi, J.L. 1998. Contribution of the autonomic nervous system to blood pressure and heart rate variability changes in early experimental hyperthyroidism. Eur. J. Pharmacol. 352(2-3): 247-255.

Senges, J., Brachmann, J., Pelzer, D., Hasslacher, C., Weihe, E., and Kubler, W. 1980. Altered cardiac automaticity and conduction in experimental diabetes mellitus. J. Mol. Cell. Cardiol. 12(12): 1341-1351.

Shi, W., Wymore, R., Yu, H., Wu, J., Wymore, R.T., Pan, Z., Robinson, R.B., Dixon, J.E., McKinnon, D., and Cohen, I.S. 1999. Distribution and prevalence of hyperpolarization-activated cation channel (HCN) mRNA expression in cardiac tissues. Circ. Res. 85(1): e1-6. 
1 Soltysinska, E., Speerschneider, T., Winther, S.V., and Thomsen, M.B. 2014. Sinoatrial node

2 dysfunction induces cardiac arrhythmias in diabetic mice. Cardiovasc. Diabetol. 13: 122. doi:

$3 \quad 10.1186 / \mathrm{s} 12933-014-0122-\mathrm{y}$.

4 Sridhar, A., Dech, S.J., Lacombe, V.A., Elton, T.S., McCune, S.A., Altschuld, R.A., and Carnes,

5 C.A. 2006. Abnormal diastolic currents in ventricular myocytes from spontaneous hypertensive

6 heart failure rats. Am. J. Physiol. Heart. Circ. Physiol. 291(5): H2192-2198. doi:

7 10.1152/ajpheart.01146.2005.

8 Stieber, J., Hofmann, F., and Ludwig, A. 2004. Pacemaker channels and sinus node arrhythmia.

9 Trends. Cardiovasc. Med. 14(1): 23-28.

10

11

12

13

Stieber, J., Wieland, K., Stockl, G., Ludwig, A., and Hofmann, F. 2006. Bradycardic and proarrhythmic properties of sinus node inhibitors. Mol. Pharmacol. 69(4): 1328-1337. doi: 10.1124/mol.105.020701.

Tsuchida, K., and Watajima, H. 1997. Potassium currents in ventricular myocytes from genetically diabetic rats. Am. J. Physiol. 273(4 Pt 1): E695-700.

Veglio, M., Chinaglia, A., and Cavallo Perin, P. 2000. The clinical utility of QT interval assessment in diabetes. Diabetes. Nutr. Metab. 13(6): 356-365.

Wang, D.W., Kiyosue, T., Shigematsu, S., and Arita, M. 1995. Abnormalities of K+ and Ca2+ currents in ventricular myocytes from rats with chronic diabetes. Am. J. Physiol. 269(4 Pt 2): H1288-1296.

Wild, S., Roglic, G., Green, A., Sicree, R., and King, H. 2004. Global prevalence of diabetes: estimates for the year 2000 and projections for 2030. Diabetes. Care. 27(5): 1047-1053.

Zhang, L., Parratt, J.R., Beastall, G.H., Pyne, N.J., and Furman, B.L. 2002. Streptozotocin diabetes 
1 protects against arrhythmias in rat isolated hearts: role of hypothyroidism. Eur. J. Pharmacol.

$2 \quad 435(2-3): 269-276$.

3

4

5

6

7

8

9

10

11

12

13

14

15

16

17

18

19

20

21

22 
1

2 Table 1. Primer pairs used in real-time quantitative RT-PCR.

\begin{tabular}{|c|c|c|c|}
\hline Gene & Gene loci & Primer sequences & Amplicon (bp) \\
\hline \multirow[t]{2}{*}{ HCN1 } & $N M-053375$ & F: 5'-ACACCCACCTGACCAGAGAAG-3' & 217 \\
\hline & & R: 5'-CCCGAGGACATCTGTCTGAAC-3' & \\
\hline \multirow[t]{2}{*}{$\mathrm{HCN} 2$} & $N M-053684$ & F: 5'-TCAACGAGGTGCTGGAGGAGT-3' & 206 \\
\hline & & R: 5'-CTCTGCCTGCTGCACCATCT-3' & \\
\hline \multirow[t]{2}{*}{ HCN3 } & $N M-053685$ & F: 5'-CGTTCCCAGGTGTCTCTGTTG-3' & 227 \\
\hline & & R: 5'-ACTGGCTCAGGCACTGATGAC-3' & \\
\hline \multirow[t]{2}{*}{ HCN4 } & NM-021658 & F: 5'-CTCCAGGAGCCTGCAGTTCTC-3' & 228 \\
\hline & & R: 5'-GCAAGAAGTGCTCCAGGAGTC-3' & \\
\hline \multirow[t]{2}{*}{$\beta$-actin } & $N M-031144$ & F:5'-GACAGGATGCAGAAGGAGATTACTG-3' & 87 \\
\hline & & R:5'-CACAGAGTACTTGCGCTCAGGA-3' & \\
\hline
\end{tabular}

$3 \quad F$, forward primer; $R$, reverse primer

4

5

6

7

8

9

10

11

12 


\section{$2 \quad$ Figure legends}

3 Figure 1. Changes of physical characteristics and SAN function in STZ-induced

4 diabetic rats. RHR, resting heart rate; IHR, intrinsic heart rate; SACT, sinoatrial

5 conduction time; CSNRTmax, rate-corrected maximal sinoatrial node recovery

6 time. ${ }^{*} p<0.05$ vs. the control; ${ }^{\#} p<0.01$ vs. the control; $n=10$.

Figure 2. Effect of ivabradine on the cycle length (CL) in Langendorff hearts from STZ-induced diabetic rats. (A) Representative electrocardiogram recorded in two groups. (B) Reduced effect of ivabradine on CL in the STZ-induced diabetic group.

Figure 3. Expression of $\mathrm{HCN}$ channel transcripts. (A and B) HE-stained sections of SAN tissues before capture. (C) The remaining tissue after laser capture of the SAN cells. Asterisks mark the SAN artery as a landmark. (D) Transcription of HCN2 and HCN4 in laser-captured SAN cells. ${ }^{*} p<0.05$ vs. the control; $n=5$.

Figure 4. Pacemaker activity and activation pattern in SAN. (A) Rat atrial preparation containing SAN used for optical mapping experiments. SVC, opening of the superior vena cava; IVC, opening of the inferior vena cava; IAS, interatrial septum; CT, crista terminalis. (B) Representative activation maps in atrial preparations from a

21 STZ-induced diabetic rat and a control. Time interval between isochrones is $1.5 \mathrm{~ms}$.

22 (C) Location of the leading pacemaker sites of the STZ-induced diabetic rats and the 
1 7 18 19 .

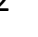
4 25

control group. Orange axes were placed adjacent to the crista terminalis and along the opening of the inferior vena cava. Blue points represent the leading pacemaker sites of the STZ-induced diabetic SAN. Yellow points represent the leading pacemaker sites of the control. (D) Comparison of the cycle length between the two groups. (E) Comparison of SAN conduction velocity between the two groups. (F) Representative optical APs from a diabetic heart and a control heart. Optical APs were measured in the region of the leading activation site in the SAN. (G) Comparison of DD slope between the two groups. (H) Comparison of APD50 and APD70 between the two group. ${ }^{*} p<0.05$ vs. the control; $\mathrm{n}=20 .{ }^{*} p<0.01$ vs. the control; $n=20$.

Figure 5. Expression of $\mathrm{HCN}$ channel proteins. (A) Confocal images of serial sections of rat SAN tissues labeled with $\mathrm{Cx} 43, \mathrm{HCN} 2$, and HCN4 from the STZ-induced diabetic rats. SNA, sinus node artery; IAS, interatrial septum; endo, endocardium; epi, epicardium. Scale bar $=300 \mu \mathrm{m}$. (B) Western blot assays of HCN2 and HCN4 for two groups. ${ }^{\#} p<0.01$ vs. the control; $n=20$. 20 
A

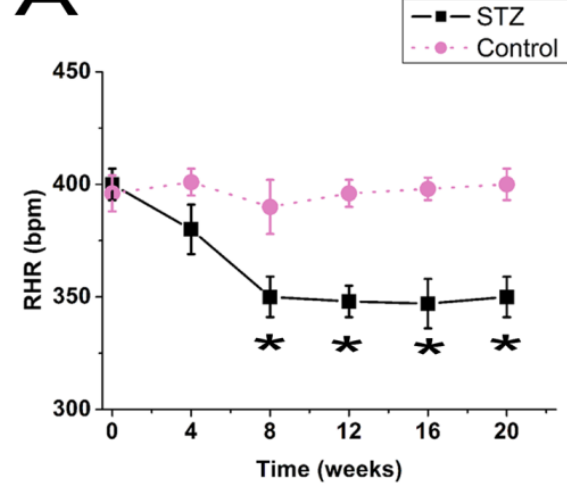

D

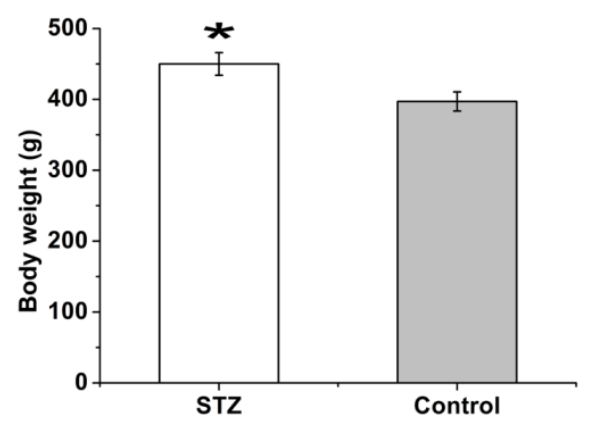

Canadian Journal of Physiology and Pharmacology

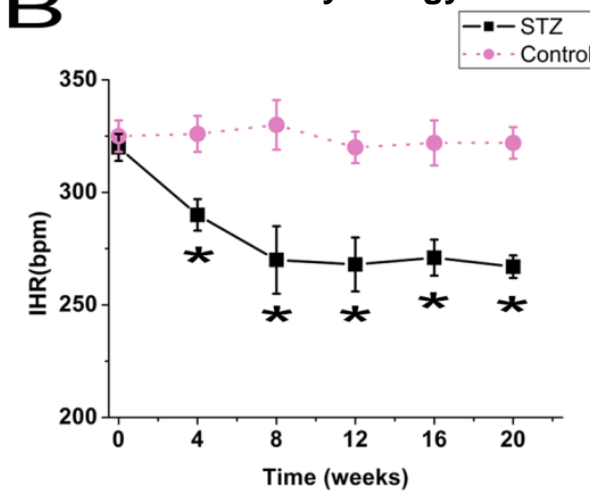

E

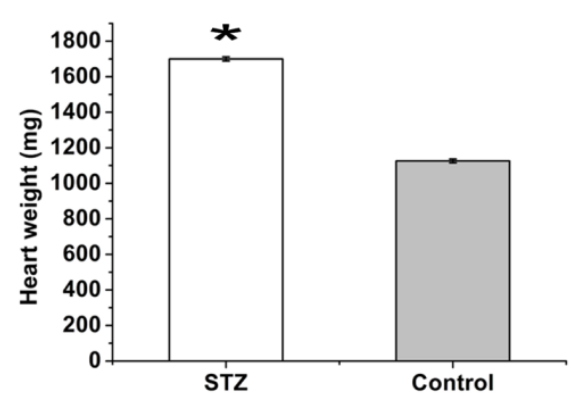

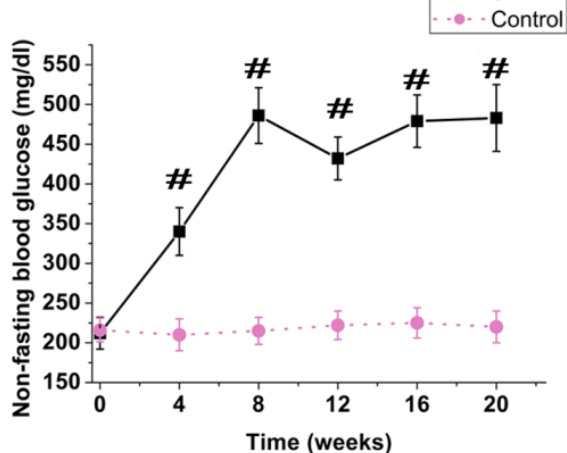

F

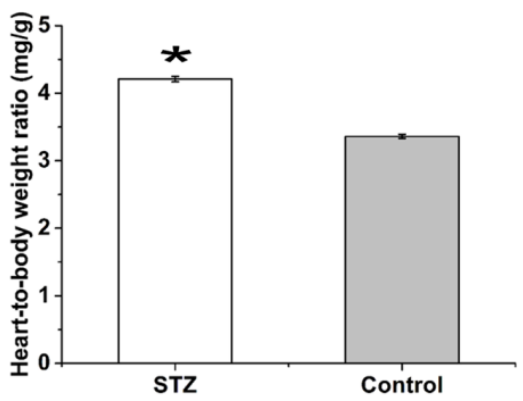

$\mathbf{G}$

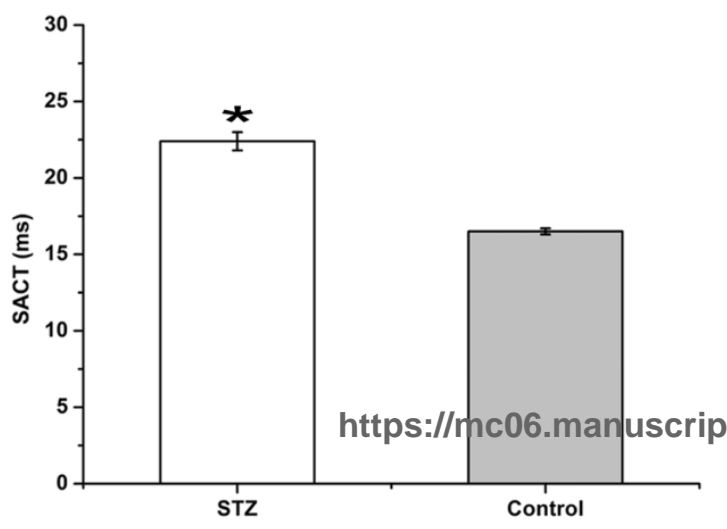

H

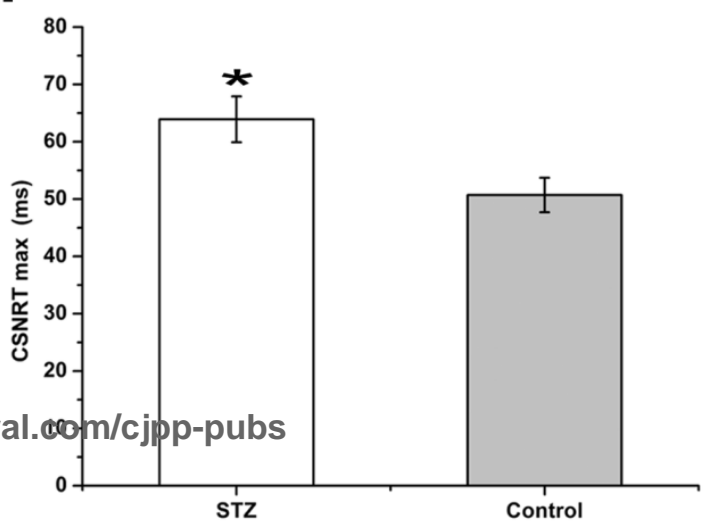


A

STZ

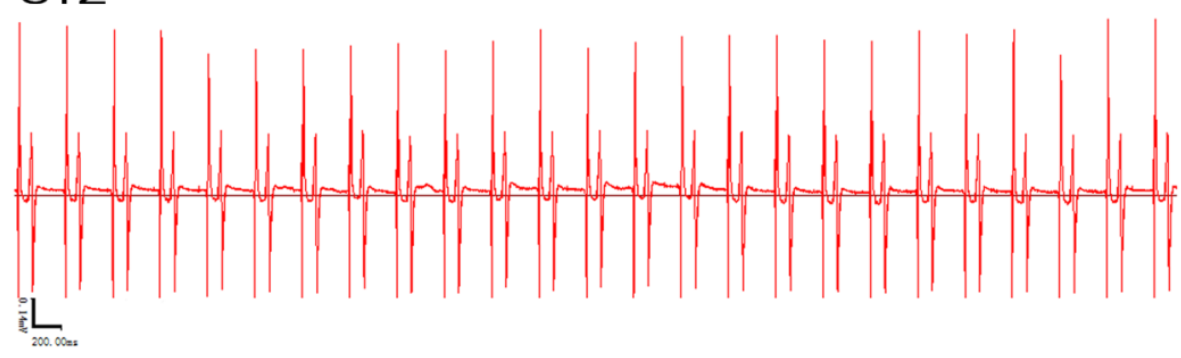

\section{Control}

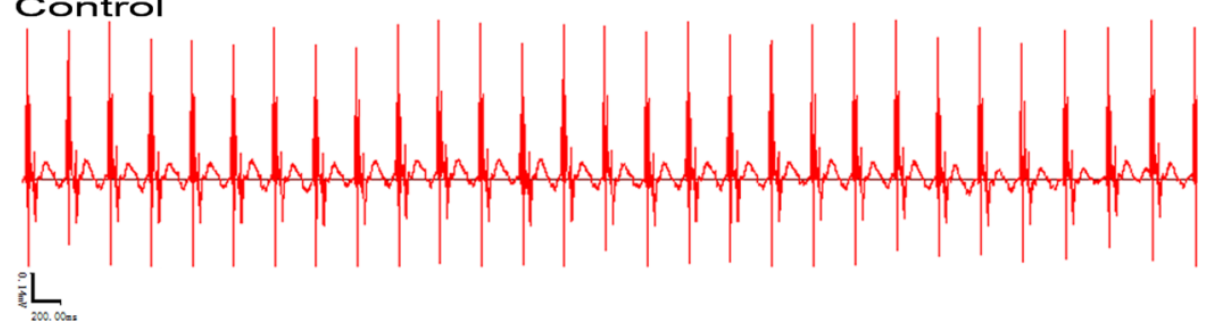

B

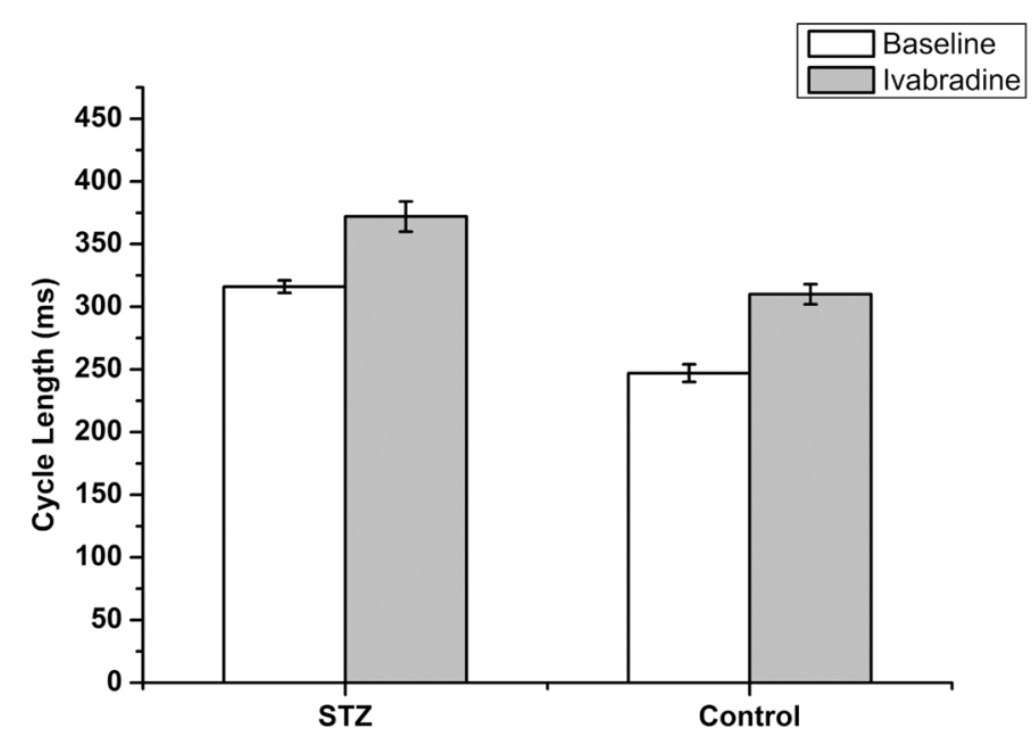



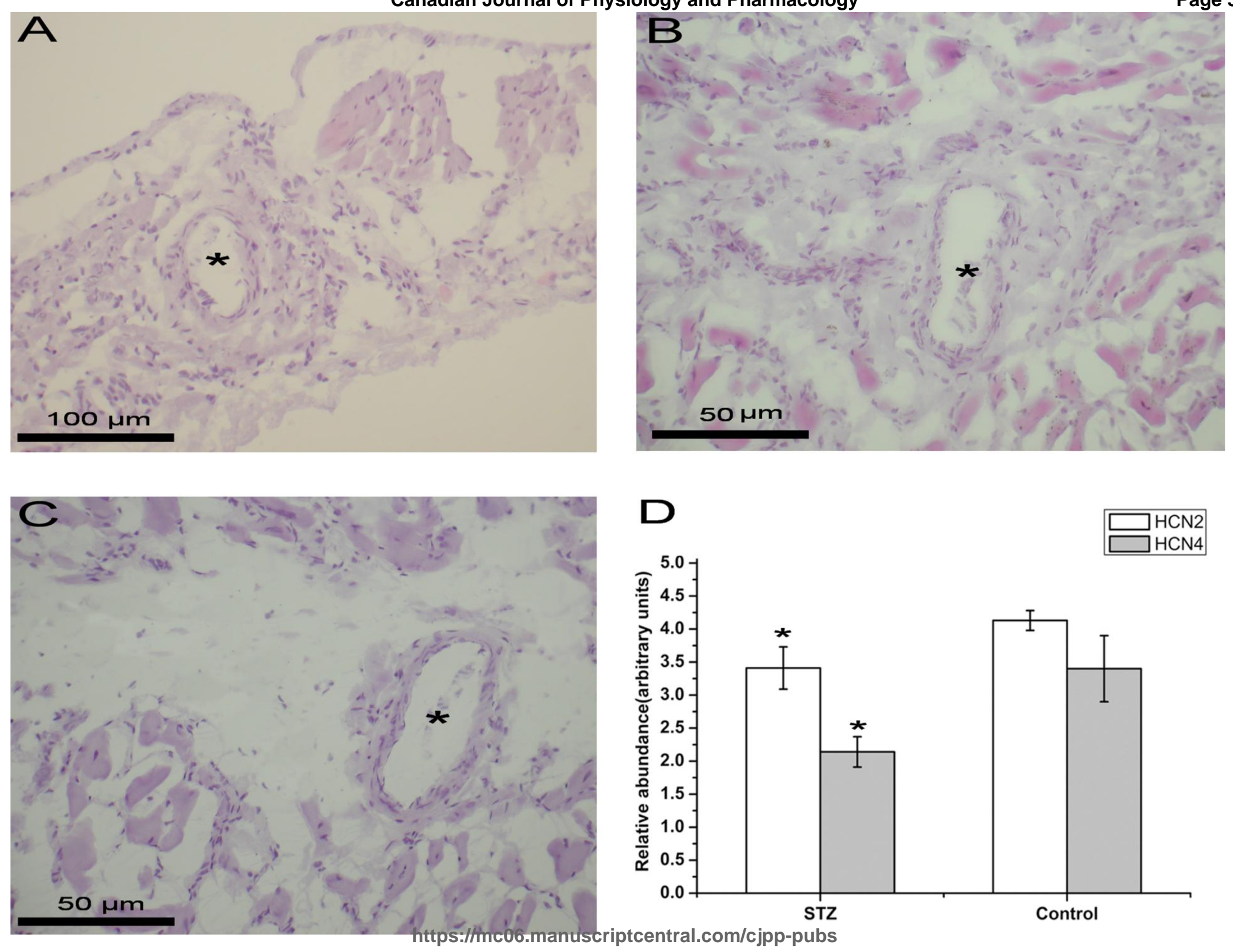


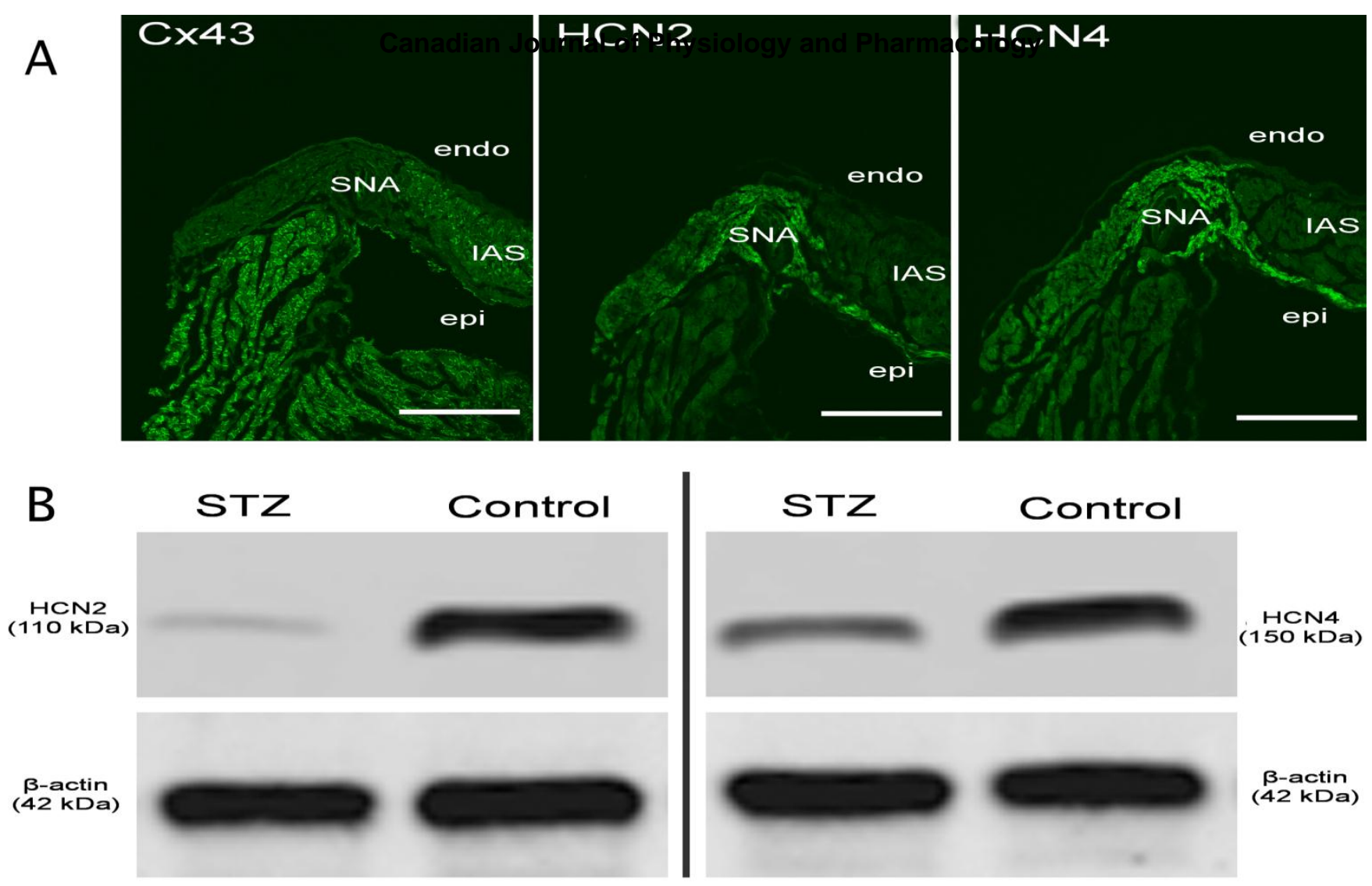

Page 32 of 32

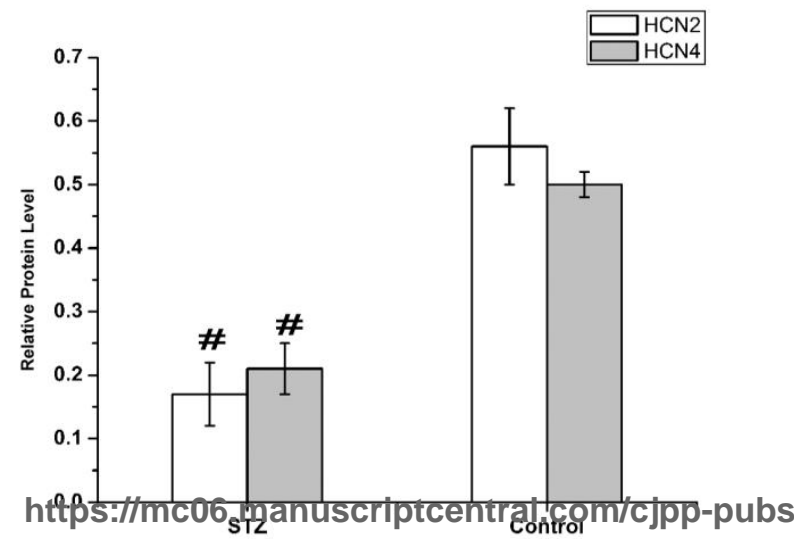

